\section{Propiedad Intelectual y Sostenibilidad: La protección de los conocimientos tradicionales}

Lucas Matías Lehtinen ${ }^{(*)}$

Resumen: Se analiza el concepto de propiedad intelectual y se armoniza con el concepto de sostenibilidad. En particular se muestra un ejemplo concreto de posible regulación: los conocimientos tradicionales y con ello, se demuestra que es posible armonizar el aprovechamiento económico de la propiedad intelectual con un impacto innovador en el desarrollo social y del medioambiente cultural.

Palabras claves: propiedad intelectual - desarrollo - conocimientos tradicionales.

[Resúmenes en inglés y portugués en las páginas 178-179]

${ }^{(*)}$ Abogado por la Universidad de Córdoba, Máster en Derecho de la Propiedad Intelectual por la Universidad Austral. Premio mentes innovadoras del Desarrollo AmCham (2010).

\title{
Introducción
}

La importancia de las prácticas comerciales para resolver los problemas actuales de sostenibilidad, como el cambio climático, la degradación ambiental y las desigualdades sociales han sido enfatizadas en la literatura durante bastante tiempo.

A partir de una perspectiva empresarial las áreas de sostenibilidad y responsabilidad corporativas buscan abordar estos problemas trascendiendo las responsabilidades centrales de las empresas a aspectos no económicos, como son sus responsabilidades sociales y ecológicas (Yunus \& Weber, 2007).

Esta noción se aborda con frecuencia como un modelo de negocio de "triple impacto", generando empresas denominadas "B" que más allá de la rentabilidad, buscan un sentido a su pertenecer a una comunidad determinada.

De esta manera, los incentivos tienden a generar y administrar el conocimiento para propender a dotar de soluciones que generen una solución sostenible y orientada por la innovación. Es así que en la economía, política e incluso en el mundo jurídico se ha reflexionado acerca de cómo asignar los recursos y bienes con los que cuenta la sociedad. 
En las democracias contemporáneas y principalmente, desde la aparición de los derechos de incidencia colectiva, siempre se ha dado un debate que trata de sortear la contraposición entre asignación de recursos y medio ambiente.

Este debate se ha planteado como un dilema ético y político, que contiene una serie de interrogantes sociales, estos son: qué valor se debe favorecer, cómo debe ser favorecido y para qué favorecerlo. Es decir, aquí no sólo interesa el medio, sino también, el fin que se persigue, sin omitir la influencia del contexto social.

En este sentido, Rawls (Rawls, 1996) nos ilustra de la siguiente manera:

Una sociedad es una asociación, más o menos autosuficiente, de personas que reconocen ciertas reglas de conducta como obligatorias en sus relaciones y en su mayoría actúan de acuerdo con ellas. Estas reglas diseñan un sistema de cooperación y aun cuando la sociedad es una empresa cooperativa para obtener ventajas mutuas, se caracteriza típicamente tanto por un conflicto como por una identidad de intereses, hay una identidad puesto que la cooperación hace posible una vida mejor para todos, que si cada uno viviera de sus propios esfuerzos. Hay un conflicto de intereses puesto que las personas no son indiferentes respecto a cómo ha de distribuirse los mayores beneficios producidos por la colaboración, ya que con el objeto de perseguir sus fines cada una de ellas prefiere una participación mayor que una menor.

De esta manera, se pueden encontrar diversas respuestas que responden a modelos sociales y éticos distintos ${ }^{1}$. Pero, la tensión vuelve al escenario cuando la noción de propiedad ingresa. Esto se debe a que la noción de propiedad contiene en si misma el debate de qué favorecer, a quienes y para qué hacerlo.

En consecuencia, esto demuestra la tensión entre la libertad y la igualdad y nos lleva a preguntar si el concepto de derecho de propiedad es el adecuado, es decir si esta colisión entre libertad e igualdad se resuelve en forma justa o injusta.

Esta dicotomía se plantea con mayor profundidad si aquello de lo que una persona se quiere apropiar es un intangible, es decir reivindicar la propiedad de una creación, una invención o una idea² ${ }^{2}$.

De este modo, parece que la brecha entre libertad e igualdad se profundiza al reclamarse la propiedad intelectual de algo, por cuanto, esta noción entra en conflicto con otros derechos, principios o valores sociales relevantes.

En la actualidad, esgrimir el concepto de propiedad intelectual implica realizar una construcción que debe analizarse desde la teoría de la justicia y principalmente, desde la noción de justicia social y desde los beneficios que su protección trae para el contexto social entendiendo que brinda la protección necesaria y los incentivos para llevar adelante los objetivos de sostenibilidad que requiere el mundo actual.

Es por ello, que en estas páginas pretendo desarrollar que la idea de propiedad intelectual, observada desde la incidencia que en ella tienen los valores de los derechos de incidencia colectiva y de los derechos humanos, adquiriendo un nuevo significado y con ello, un nuevo contenido esencial, no ya simplemente negativo; por el contrario, con un valor positivo denominado ius innovationis, demostrando que existe una armonización 
entre propiedad intelectual y el concepto de sostenibilidad, siendo un ejemplo de ello, los conocimientos tradicionales.

\section{El fundamento de la propiedad intelectual como promotora de derechos}

Se ha dicho -Víctor Hugo- que no son las locomotoras, sino las ideas las que arrastran e impulsan al mundo. Justamente, una idea puede cambiar todo, modificar la perspectiva, darnos un nuevo camino y horizonte, impulsar una industria y revolucionar la economía. En definitiva, crear una nueva realidad, pasando desde algo intangible a lo tangible, contribuyendo a la riqueza y economía.

Cuando nos referimos a las ideas, hablamos de creaciones, de innovación y con ello, inmediatamente de propiedad intelectual. Es decir, la forma de proteger esas creaciones de la mente e invenciones, así como el arte, la literatura, el software, los símbolos, marcas, nombres e imágenes utilizadas en el comercio para generar desarrollo económico.

En consecuencia, el término propiedad intelectual se refiere a un amplio grupo de leyes que regulan el uso de diferentes tipos de creaciones. La ley de derecho de autor protege varias formas originales de expresión, incluyendo novelas, películas, composiciones musicales y programas de software. Las leyes de patentes protegen las invenciones. Las leyes de marca protegen las palabras y los símbolos identificatorios que les permiten a los consumidores distinguir los bienes y servicios manufacturados o provistos por personas o firmas particulares. Las leyes de secreto industrial protegen la información comercialmente valiosa, que las empresas intentan ocultar a sus competidores. El derecho a la imagen protege los intereses de las celebridades en el uso de sus imágenes y su identidad. En este contexto, las estadísticas de organismo internacionales, como la Organización Mundial de la Propiedad Intelectual (OMPI), muestran que hasta el 80 por ciento del incremento del producto interno bruto de los países en desarrollo, durante los últimos años, se debe a la innovación y el uso efectivo de la propiedad intelectual.

No obstante, tradicionalmente se ha entendido a la propiedad intelectual con un contenido negativo, definida como un derecho de excluir a los demás, respondiendo a uno de los significados que se ha asignado a la idea de propiedad.

Sin embargo, siguiendo la idea de primer motor, esto es lo que pone en movimiento otros elementos, podemos decir que la propiedad intelectual es el primer motor de muchos derechos y permite en su desarrollo realizar el catálogo de algunos de los derechos humanos.

Pensemos en el sistema de patentes, en particular en la industria farmacéutica, la protección de la propiedad intelectual redunda en un beneficio a la salud, generando nuevas vacunas. Es cierto, que, desde la teoría economicista, podrían establecer que sólo los individuos buscarán patentes, en sectores de la industria farmacéutica, de los que puedan obtener réditos.

Ahora bien, si sólo pensáramos desde el punto de vista de los incentivos, olvidaríamos que en realidad la protección de la propiedad intelectual genera los herramentales necesarios para el ejercicio efectivo de otros derechos fundamentales. 
En definitiva, entender el carácter instrumental de la innovación y su protección por medio de la propiedad intelectual, nos hace ver que más allá del contenido negativo que tienen estos derechos, se expresan positivamente para el conjunto social estableciendo canales que permiten realizar bienes jurídicos básicos o protegerlos.

Por ello, siguiendo el pensamiento de Sandel (Sandel, 1982), la propiedad intelectual puede verse en el marco de la teoría de la justicia y en particular, de la justicia distributiva, garantizando derechos, siendo sustento de un contenido que viene a realizar los elementos necesarios para alcanzar la felicidad humana.

Actualmente, la manifestación del componente creativo del ser humano se realiza por medio de la innovación y esta responde a los valores sociales relevantes de un tiempo y de un lugar.

Quizás el ser humano haya reflexionado -al igual que lo hizo al momento de la finalización de las grandes guerras-, a la luz de problemas como la extrema pobreza, que los problemas más acuciantes generados por un pensar utilitario extremo sean que no se puede realizar la dignidad del hombre.

Es por ello, que, en este marco, la innovación direccionada por esos nuevos valores sociales pueda entender que la propiedad intelectual en vez de un derecho a prohibir sea la benefactora de un derecho a hacer y su estímulo permita construir una regla maximizadora de la justicia y armonizar las tensiones entre libertad e igualdad.

De este modo, la teoría de la justicia aporta una visión que permite acrecentar la idea de que la innovación nutre a la propiedad intelectual con un contenido positivo, propio de otros derechos humanos, por cuanto se encuentra destinado al desarrollo de las potencialidades humanas.

En este orden de ideas, aparece el concepto de sustentabilidad orientada a la innovación, la cual permite unificar y encontrar la justificación de que la propiedad intelectual con sus incentivos puede favorecer el desarrollo sostenible y las creaciones orientadas a valores sociales.

Quizás, como señala Peter Watson, la lección más importante que podemos extraer de una historia de las ideas: que la vida intelectual -acaso la dimensión más importante, satisfactoria y característica de la existencia humana- es una cosa frágil, que puede perderse o destruirse con facilidad, sino la cuidamos y generamos la conciencia de que ella contribuye al desarrollo y progreso social.

Por consiguiente, profundizaremos en el concepto de desarrollo orientado por la innovación para entender las funciones que aporta la propiedad intelectual al desarrollo. No sin antes indagar en el fenómeno de los conocimientos tradicionales.

\section{Los conocimientos tradicionales como fenómeno protegible}

La construcción de la protección de los conocimientos tradicionales implica el resguardo de situaciones, elementos o cosas susceptibles de ser protegidas jurídicamente. Ahora bien, para ello es preciso destacar que la Secretaría de la $\mathrm{OMPI}^{3}$ ha utilizado el término "conocimientos tradicionales" de manera flexible para referirse a invenciones científicas; 
diseños; marcas; nombres y símbolos; información no divulgada y todas las demás innovaciones y creaciones basadas en la tradición resultantes de la actividad intelectual realizada en el ámbito industrial, científico, literario o artístico.

La expresión "basadas en la tradición" se refiere a los sistemas de conocimientos, creaciones, innovaciones y expresiones de sus titulares, por lo que su conservación e integridad están relacionados con la preocupación relativa a la conservación de las distintas culturas en sí; y aunque contengan información de carácter práctico o tecnológico, los conocimientos tradicionales tienen una dimensión cultural y un contexto social que los diferencia de otras formas de información científica o tecnológica.

No obstante, la experiencia recabada en el desarrollo de los conocimientos tradicionales, su mejora en base a la experiencia intergeneracional hace que preservarlos no sólo refiere a una finalidad cultural, sino a la preservación de un conglomerado de saberes y conocimientos.

Es por eso, que se procura establecer los derechos de las comunidades originarias y pueblos indígenas, como también formas de protección contra su apropiación; respondiendo a una doble finalidad, conservar la cultura ancestral y evitar la apropiación indebida de esos conocimientos.

Debido a que su creación, conservación y difusión está basada en las tradiciones culturales, los conocimientos tradicionales están fundamentalmente orientados hacia la cultura o tienen cierta predisposición hacia la cultura, y son parte integral de la identidad cultural del grupo social en el cual existen y se conservan.

Así, a diferencia del conocimiento científico que goza de un alcance universal; los conocimientos tradicionales tienden a conservar la raigambre cultural de las poblaciones locales, siendo el reflejo de su idiosincrasia y exponiendo los localismos en un mundo globalizado. De este modo, si bien tanto el conocimiento tradicional y el conocimiento científico se encuentran orientados a la cultura; el primero, se traduce en la vida cultural como un símbolo de ética y forma de vida de la comunidad.

Desde el punto de vista de la cultura de la comunidad en la que se han originado, cada componente de los conocimientos tradicionales puede ayudar a definir la propia identidad de la comunidad. Esta característica puede parecer obvia en lo que atañe a las expresiones del folclore y las artesanías, pero también se aplica a otras esferas de los conocimientos tradicionales, como son los conocimientos de medicina y agricultura. Las nociones medicinales derivadas de una cierta combinación de plantas por una comunidad sudamericana, por ejemplo, difieren necesariamente de las nociones desarrolladas por una comunidad africana sobre la base de plantas similares. Ello se debe a que el desarrollo de conocimientos medicinales por las comunidades tradicionales no sólo responde a una necesidad específica, sino también, a enfoques y creencias culturales.

Esto contrasta fuertemente con el caso de dos invenciones científicas realizadas por dos diferentes equipos de inventores empleados con el objetivo de resolver el mismo problema técnico: no es raro que las dos invenciones sean muy similares, lo que en derecho de patentes puede dar lugar a procedimientos de interferencia o procedimientos judiciales similares que adjudican la titularidad a uno u otro de los solicitantes. Las reivindicaciones de patentes competidoras con respecto a la materia duplicada se resuelven sin hacer referencia al entorno cultural que dio origen a las invenciones. 
En cambio, la dimensión de la identidad cultural de los conocimientos tradicionales puede tener un impacto espectacular en cualquier marco jurídico futuro para su protección, porque al ser un medio de identificación cultural, la protección de los conocimientos tradicionales, incluidos los conocimientos tradicionales de naturaleza técnica, deja de ser meramente una cuestión de economía o de derechos exclusivos sobre la tecnología como tal. Adquiere realmente una dimensión de derechos humanos, pues se entrelaza con cuestiones relacionadas con la identificación cultural y la dignidad de las comunidades tradicionales. Se puede establecer una analogía con el concepto de "derechos morales" del derecho de autor, concretamente con los derechos de integridad y de paternidad, en el sentido de que podría considerarse necesaria una protección contra el uso culturalmente ofensivo de los conocimientos tradicionales u otras formas no económicas de uso indebido de los conocimientos tradicionales.

La forma en que se crean las innovaciones puede obedecer a reglas basadas en la cultura. Sin embargo, la manera en que se crean los conocimientos tradicionales puede parecer, desde una perspectiva externa o universal, como no sistemática y sin método, en parte porque el reglamento o sistema que rige su creación puede transmitirse de manera informal, en parte porque este elemento sistemático no está explícitamente articulado, y en parte porque el proceso que lleva a la creación de los conocimientos tradicionales puede no estar formalmente documentado de la manera en que se registra la mayoría de la información científica y tecnológica.

Así, la forma aparentemente no sistemática de creación de los conocimientos tradicionales no disminuye su valor cultural o su valor desde el punto de vista de los beneficios técnicos, y plantea la pregunta de cómo registrar o definir la relación que guardan con el sistema de conocimientos, conjunto de normas o directrices culturalmente específicos, o con las creencias básicas que ayudan a formarlos.

$\mathrm{Al}$ igual que con la característica "basada en la tradición", la característica aparentemente "no formal" hace que se ponga especial énfasis en el contexto de creación, y en la necesidad potencial de que se consideren los elementos de este contexto cultural junto con los conocimientos en sí.

No obstante, cabe remarcar que muchas veces se refiere con el término de conocimientos tradicionales casi de forma exclusiva a los conocimientos de los pueblos indígenas. Aunque, no son los conocimientos indígenas los únicos conocimientos tradicionales; si bien son la especie más representativa e importante de conocimientos tradicionales.

De esta manera, muchas comunidades rurales y campesinas, aledañas a un ecosistema, pueden tener algún conocimiento derivado de alguna práctica de la biodiversidad, como son: pescadores, ecologistas, mineros, habitantes de zonas geográfica o climáticas especiales, entre otros.

Así, cabe diferenciar el concepto genérico de comunidad originaria, como comprensivo del concepto de pueblo indígena. De este modo, todo pueblo indígena es una comunidad originaria, pero no toda comunidad originaria es un pueblo indígena.

Nakayima explica que el conocimiento tradicional no es únicamente pasado de generación en generación, sino que además, por ser dinámico, es objeto de un continuo proceso de verificación, adaptación y creación, alterando su forma y contenido en respuesta a 
los cambios ambientales y las circunstancias sociales. Tendríamos entonces, que afirmar, que cuando hablamos de "conocimiento tradicional" a lo que hacemos alusión es a conocimientos dinámicos, basados en valores milenarios de los pueblos indígenas. Lo que determina que se expresen formalmente y se destaque su posibilidad de otorgar una protección distinta de la tradicionalmente otorgada a la propiedad intelectual.

De esta manera, la naturaleza jurídica de los conocimientos tradicionales, los cuales exceden el mero ámbito de protección negativo de los Derechos de Propiedad Intelectual y presentan una concepción teleológica distinta, puesto que tienen una naturaleza sui generis y esto se refleja en: a) Su durabilidad temporal; b) Su conformación histórica y afincada en las raíces culturales de la comunidad que le dio origen; c) Su transmisión de generación en generación; d) Su representación como forma de vida del pueblo ${ }^{4}$. Todo esto hace confluir en que los conocimientos tradicionales tienen una salvaguarda y protección especial, por cuanto no se protege sólo la imposibilidad de accionar de terceros -cuestión que comparten con los Derechos Intelectuales (Ius-Prohibendi)- sino la idiosincrasia del pueblo al cual acceden.

\section{El contexto internacional de protección}

Actualmente, no existe un marco regulatorio en varios países y existen diversos instrumentos internacionales ${ }^{5}$ que reconocen derechos a los pueblos indígenas, alguno de ellos incluso sobre la propiedad intelectual de sus creaciones ${ }^{6}$.

Así, en el año 2002, la Conferencia de las partes del CDB adoptó las directrices de Bonn sobre acceso a los recursos genéticos y distribución justa y equitativa de los beneficios derivados de su utilización, en su sexta reunión, en la Haya.

Estas directrices voluntarias guían tanto a los proveedores, como a los usuarios, de recursos genéticos en la aplicación de las disposiciones del Convenio acerca del acceso y la participación en los beneficios.

Las directrices fueron adoptadas para ayudar a las partes a establecer medidas administrativas, legislativas o de política sobre acceso y participación de los beneficios y/o a los proveedores y usuarios a negociar acuerdos para el acceso a los recursos genéticos y la participación en los beneficios.

El protocolo de Nagoya sobre acceso a los recursos genéticos y participación justa y equitativa en los beneficios que se deriven de su utilización al convenio sobre la diversidad biológica fue adoptado por la Conferencia de las partes en su décima reunión, en Nagoya, Japón, el 29 de octubre de 2010. Desde allí, se remarcaron la consagración de que los tres principios: a) acceso, b) participación en los beneficios y c) consentimiento previo; mantienen una unión indisoluble.

De este modo, el Convenio de Diversidad Biológica establece que una persona o institución que desee acceder a recursos genéticos en un país extranjero deberá obtener el consentimiento fundamentado previo del país donde se encuentra dicho recurso. Es más, esa persona o institución también deberá negociar y acordar las condiciones de acceso 
y utilización del recurso, incluida la distribución de los beneficios que se deriven de la utilización de dicho recurso con el proveedor como un requisito previo para el acceso al recurso genético y la utilización de este.

A su vez, los países, cuando actúan como proveedores de recursos genéticos, deberán crear las condiciones necesarias para facilitar el acceso a sus recursos genéticos para utilizaciones ambientalmente adecuadas y no impondrán restricciones contrarias a los objetivos del Convenio de Diversidad Biológica.

En este sentido, la consecución de instrumentos internacionales y los esfuerzos de las diversas organizaciones han tendido a transparentar el sistema de acceso y uso de los recursos genéticos y conocimientos tradicionales.

No obstante, se presentan nuevos desafíos puesto que los avances tecnológicos permiten flexibilizar aún más los controles del acceso, evitando que se sigan los procedimientos tangibles diseñados por los ordenamientos jurídicos internacionales, tendiendo a crear una supuesta virtualidad de la vigencia de la ley, frente a la conculcación sistemática de los derechos, en virtud de estos avances.

\section{Los desafíos de los cambios tecnológicos}

Desde que el Convenio sobre la Diversidad Biológica reconociera la soberanía de los Estados sobre sus recursos genéticos, y los mismos fueran definidos, los cambios tecnológicos en el campo de las Ciencias Biológicas han sido abundantes. Estos cambios implican alteraciones en la gestión del acceso a los recursos biológicos y genéticos.

Entre estos cambios podemos señalar:

- La proteómica es el estudio a gran escala de las proteínas, sus estructuras y sus funciones. Esta disciplina está dirigida a estudiar específicamente las proteínas que se generan del ADN y el ARN y "su meta es realizar una descripción comprehensiva y cuantitativa de las expresiones de las proteínas y sus cambios bajo la influencia de perturbaciones biológicas como enfermedades o terapias farmacológicas".

- La bioinformática hace referencia a la aplicación de las ciencias de la computación y de las tecnologías de la información al campo de estudio de la biología, y de la biología molecular en particular. Ella ha probado ser una herramienta crucial para la genómica y para el secuenciamiento del ADN de varios organismos, así como para la proteómica. Al aplicar las tecnologías de la información, la humanidad puede expandir y desarrollar el entendimiento de los procesos biológicos mucho más allá de lo esperado. En la práctica, la bioinformática es un modo de llegar a conocer el valor en el material genético, desconectado de las fuentes biológicas donde fue, originalmente, hallado.

- La biología sintética que permite extraer de sistemas vivos partes intercambiables que pueden ser probadas, validadas como unidades de construcción, y posteriormente reconstruidas para crear aparatos que pueden (o no) tener un sistema vivo análogo o similar. Sí cuando el conocimiento y la tecnología hacen posible recrear la biología en tales formas de forma segura y precisa, el uso del material genético microfísico puede ser relegado a una posición remota. Sin embargo, en la medida que la biología sintética 
copia la biología de una manera sintética, es altamente probable que haya habido algún uso del material genético en el proceso. Además, en la biología sintética, la conexión con el material genético se vuelve más distante. Este es un modo de conocer el real valor de los aspectos informativos de las unidades funcionales de herencia.

Todos estos cambios permiten esbozar la teoría de que es inverosímil intentar proteger y otorgar un régimen de reconocimiento a los conocimientos tradicionales, por cuanto esto implicaría formas de protecciones anticuadas y analógicas en una era digital. Estableciendo un estándar que haría obsoleto cualquier régimen de protección basado en las regulaciones del actual marco normativo.

Sin embargo, estas aseveraciones distan de reconocer que el desconocimiento legislativo por parte de los Estados a consagrar esta protección provocaría un vacío que permitiría la proliferación y el escape de la riqueza de los ecosistemas naturales y su migración transfronteriza, con la pérdida de recursos económicos valiosos y peor aún con la degradación cultural de las tradiciones de los pueblos y comunidades originarias.

Es importante destacar que pese a estos avances se requiere la consagración legislativa de un régimen de protección de los conocimientos tradicionales que reconozca un mínimo de protección para transparentar el sistema de patentes.

Por consiguiente, se pone de manifiesto la posibilidad de incluirlos para el análisis del estado del arte en el procedimiento de patentes, como también la denuncia del origen del conocimiento tradicional asociado a una invención. Todo eso permite equilibrar y establecer las condiciones de convivencia del régimen de propiedad intelectual y los conocimientos tradicionales.

Así, el concepto de acceso sin limitaciones ha ido cambiando, primero, con el Compromiso Internacional de Recursos Fitogenéticos - de la FAO- que establece el derecho soberano a dichos recursos, en los artículos 5, 6, 10 y 12 y luego con la Convención sobre Diversidad Biológica, que, en su artículo 15, reconoce "el derecho soberano de los Estados sobre sus recursos naturales, la facultad de regular el acceso a los recursos genéticos incumbe a los gobiernos nacionales y está sometida a la legislación nacional”.

De este modo, de la redacción surge que los Estados son los dueños de los recursos naturales en su territorio, las interpretaciones que se han dado de este artículo van más en la línea de interpretar que el Estado tiene autoridad única y exclusiva para regular el acceso a los recursos genéticos.

De hecho, si se reconoce que lo que el Estado tiene es la potestad para determinar las formas de acceso a los recursos biológicos, entonces este mismo Estado puede establecer mecanismos a través de los cuales se respeten los derechos de los pueblos indígenas a decidir sobre sus recursos y CCTT asociados; es decir, a ser autónomos en este sentido, sin tener necesariamente que entrar en una confrontación con el Estado.

Algunas legislaciones, como las de Costa Rica, Panamá, Brasil, Filipinas, Bután, entre otras, prevén el consentimiento fundamentado previo por parte de los pueblos indígenas antes de que el Estado autorice un contrato de acceso, rescatando el derecho de los pueblos indígenas a negarse a tal acceso. Es decir, existe un doble control de los recursos y CCTT asociados con respecto a las decisiones de los pueblos indígenas.

En efecto, los tiempos en que los recursos biológicos eran considerados "patrimonio común" quedó en la historia, y en la actualidad tenemos que guiarnos por el concepto 
de "interés común" de la humanidad, que, más ni menos, es el interés internacional en la conservación de la diversidad biológica, aunque este pertenezca a diferentes Estados y esté regulada por distintas leyes.

Por ello, los conocimientos tradicionales al estar asociados a los recursos genéticos y biológicos forman parte de la diversidad biológica, integran el concepto de interés común de la humanidad, puesto que se garantizan los derechos de sus titulares y la protección de estos conocimientos para evitar, con su utilización indebida, una incidencia indirecta en la degradación del ambiente y su diversidad.

De este modo, la potenciación de los titulares de los conocimientos tradicionales puede ser considerada como un aspecto de derechos humanos de la protección de los derechos tradicionales. Aunque esta cuestión tiene dos componentes adicionales que no dejan de ser importantes. Uno es de naturaleza jurídica: un sistema claro, transparente y eficaz de protección de los conocimientos tradicionales aumenta la seguridad jurídica y la previsibilidad para beneficio no sólo de los titulares de los conocimientos tradicionales sino también de la sociedad en su conjunto, incluidas las empresas e instituciones de investigación dedicadas a la bioprospección. El otro es de naturaleza económica: el formalizar y registrar los activos intangibles de las comunidades tradicionales los transformaría en capital, lo cual permitiría a las comunidades tradicionales realizar operaciones comerciales de manera más segura.

Muchas comunidades tradicionales que viven en la pobreza son en realidad ricas en conocimientos, pero al no ser sus conocimientos objeto de títulos de propiedad en buena y debida forma, son susceptibles de apropiación comercial indebida por parte de terceros.

\section{La supuesta tragedia de los anti-commons}

El aspecto fundamental de la propiedad intelectual es que abarca los activos intangibles y que confiere a los titulares el derecho de prohibir que otros reproduzcan su obra y/o fijen sus interpretaciones o ejecuciones o las reproduzcan (derecho de autor y derechos conexos) así como el derecho de excluir a otros de la utilización del objeto protegido (derechos de propiedad industrial). La idea fundamental es que la propiedad intelectual es el derecho de decir "no" a terceros (y en consecuencia, el derecho de decir "si" a una persona que solicite el permiso de reproducir y/o utilizar el objeto protegido).

En consecuencia, la utilización y conservación de la biodiversidad se ordenan con regímenes de propiedad que parecen expresar la influyente tesis de Hardin (Hardin, 1968) acerca de la tragedia de los bienes comunes. Una de las ideas centrales en esta teoría es, precisamente, que los bienes sobre los que no existen derechos de propiedad definidos y precisos están destinados irremediablemente a la ruina. Esta tesis se basa en un modelo bajo el cual los usuarios de un bien estarían inclinados a obtener el mayor provecho individual en detrimento de los demás beneficiarios. Conforme a la predicción de esta teoría, los bienes de libre acceso se destruirán a menos que se establezcan derechos de propiedad individual o que el Estado intervenga coactivamente.

Sin embargo, el análisis de sistemas de propiedad comunal existentes ha dejado en evi- 
dencia algunas de las limitaciones del modelo antes descripto. Varios estudios ${ }^{7}$ han mostrado que los grupos humanos de sociedades tradicionales -pueblos indígenas y comunidades originarias- desarrollan instituciones colectivas solidarias y mecanismos de sanción social que no sólo previenen la ruina de los bienes comunes, sino que fundamentan el uso sostenible de los recursos naturales bajo regímenes de propiedad común. La tesis de Hardin tampoco podría generalizarse a las comunidades locales vinculadas culturalmente con su territorio ancestral.

Antes que depredación, los vínculos con el territorio y con los recursos biofísicos por parte de las comunidades que conservan, en lo esencial, su cosmovisión e integridad cultural, por la cual promueven relaciones de respeto hacia la naturaleza y de solidaridad hacia los demás miembros de la comunidad.

Por ello, se establece la continuidad generacional en el uso de los conocimientos tradicionales y la posibilidad de una excepción de uso intergeneracional de estos conocimientos, como una representación de esa cosmovisión e integridad que representan los conocimientos tradicionales para sus titulares.

Si se desarrollan de manera acertada, los sistemas de propiedad intelectual pueden llegar a desempeñar una función esencial en la conservación de la identidad cultural de las comunidades tradicionales, y, por consiguiente, en la potenciación de los titulares de conocimientos tradicionales, en el sentido de que se les atribuirá el derecho vital de decir "no" a terceros que utilicen sus conocimientos tradicionales sin autorización o distorsionando su uso, sea cual fuere su naturaleza comercial.

No obstante, se postula que el exceso de protección en el ordenamiento puede traer aparejado un desincentivo a la producción de innovación y con ello, trabas burocráticas al desarrollo y crecimiento de la producción de intangibles.

Esta teoría conocida como tragedia de los anti-commons. La teoría de Heller ha resultado muy útil en otras áreas del Derecho. Así, tanto Heller y Eisenberg consideran que la existencia de innumerables derechos de Propiedad Industrial, paradójicamente, perjudica las posibilidades de mayor innovación. Estos autores (Heller y Eisenberg, 1998) consideran que "la reciente proliferación de derechos intelectuales en la investigación biomédica sugiere una tragedia diferente (...) en la que las personas hacen un uso muy limitado de recursos escasos porque demasiados titulares pueden bloquearse mutuamente". En ese sentido, los innumerables derechos de exclusiva otorgados actuarían como barreras a la innovación, desnaturalizando los objetivos del Derecho de Patentes.

Una cantidad elevada de derechos de exclusión en el campo del desarrollo tecnológico puede evitar mayor investigación porque quien desea investigar (i) puede no tener claro si su investigación afectará los derechos de un tercero y (ii) si tiene claro que su investigación choca con los derechos vigentes de terceros es posible que necesite pedir demasiadas licencias para comercializar su invento y, esta situación, puede desalentarlo. Sin embargo, sostener que el reconocimiento de un derecho subjetivo puede generar un obstáculo, implicaría desconocer los ámbitos de protección de las normas. En este sentido cabe destacar que la consecuencia señalada por Heller se logra si no se otorga la dinámica correcta a cada derecho y se armonizan los intereses en juego.

Por tanto, reconocer un sistema de protección a los conocimientos tradicionales, implicaría no generar un obstáculo burocrático a la producción de innovación, por el con- 
trario, su ámbito formal sólo procura en un contenido mínimo asegurar transparencia en un sistema de reivindicaciones que en su ejercicio va más allá del ámbito formal del derecho reconocido, extendiéndose a su esfera material.

Buitrago (Buitrago, 2017) señala que la economía post industrial se sustenta en dos pilares básicos, el conocimiento y la información, esto es así para todos los campos del conocimiento científico. Estos pilares conforman los activos intangibles de instituciones y empresas, adquiriendo la condición de bien o mercancía y, como tal, valor económico. Asimismo, ejemplifica -Buitrago (Buitrago, 2017)- estableciendo que la biotecnología como motor de desarrollo profundiza estas características con sus desarrollos científico-tecnológicos y con el impacto que estos están generando en todos los campos del desempeño humano y la economía, como el medio ambiente, la salud, la agricultura, el pecuario, alimentos, industrial, entre otros.

\section{La relación entre desarrollo sostenible, cultura y propiedad intelectual}

De este modo, la necesidad de un entendimiento común de la relación entre el Convenio sobre la Diversidad Biológica, el Protocolo de Nagoya, entre otros instrumentos internacionales y el ADPIC en los distintos foros institucionales y el deber jurídico de lograr que se refuercen mutuamente como herramientas del desarrollo sostenible parten, evidentemente, de la aceptación de que los regímenes de la biodiversidad y la propiedad intelectual están, de alguna manera, relacionados entre sí.

En este sentido, examinamos la relación de los derechos de propiedad intelectual con cuatro aspectos materiales del régimen de la biodiversidad: 1) la conservación y utilización sostenible de la biodiversidad; 2) el acceso a los recursos genéticos, que comprende las interacciones negativas que supone la "biopiratería" pero también las positivas de la bioprospección; 3) las transferencias de tecnología y 4) los conocimientos tradicionales; los cuales difícilmente encuentran cabida en el actual régimen de la propiedad intelectual, lo que supone un obstáculo para el desarrollo sostenible ${ }^{8}$.

Este último aspecto material, se correlaciona con los anteriores y los representa; puesto que, en los conocimientos tradicionales, existe el origen y la causa eficiente de muchos aprovechamientos de la biodiversidad, de los recursos genéticos y de transferencias de tecnologías; que tienen implicancias económicas y productivas, las cuales exceden el marco de una economía fordista y nos muestra la importancia del valor mercantil de los intangibles.

De esta manera, se ha puesto de manifiesto que las lagunas legislativas existentes en esta materia permiten generar "incentivos perversos" conforme han sido reconocidos por la Secretaría Ejecutiva del Convenio de Diversidad Biológica, los cuales trasuntan en aprovechamientos indebidos de conocimientos cuyas "invenciones" son públicas y notorias, representativas de la colectividad de destino que forman las comunidades tradicionales. Sin embargo, cabe destacar que los derechos de la propiedad intelectual no pueden atentar, por sí solos ${ }^{9}$, contra la conservación y el uso sostenible de la diversidad biológica; incluso, en algunos aspectos, contribuyen al mejoramiento de las condiciones de vida; 
pero, no por ello, debe dejarse de lado que es necesario, concertar un reconocimiento al origen de los conocimientos tradicionales; a los fines de asegurar una mejor distribución de los recursos y el reconocimiento no sólo económico, que corrija los subsidios indirectos entre productores de conocimientos tradicionales y sus "detentadores" formales de derechos intelectuales; sino, por sobre todo un reconocimiento cultural, histórico y social de preexistencia de los mismos ante cualquier solicitud formal de apropiación.

\section{La noción de innovación orientada a la sostenibilidad}

El criterio consistente en orientar las actividades innovadoras al logro de determinados objetivos tecnológicos y sociales no es novedoso. Los programas de innovación orientada a misiones concretas suelen buscar soluciones que se consideran no atendidas por los mercados. Esos programas actúan mediante redes a escala nacional o internacional, así como a través de estructuras de incentivos.

Sin embargo, la justificación de la innovación orientada a misiones concretas cayó en desgracia en los decenios de 1980 y 1990 (Mazzucato, 2013), como reflejo de la preocupación por la incapacidad de elegir a ganadores y la opinión de que los Gobiernos solo deben actuar ante los fallos del mercado.

No obstante, más recientemente, la atención y la amplia gama de inversiones centradas en la innovación orientada a misiones concretas ha reflejado un debilitamiento de los principios tradicionales orientados a los mercados y el hincapié en los denominados grandes problemas de la sociedad (como el cambio climático, el envejecimiento, las desigualdades y las enfermedades crónicas e infecciosas). Algunos de esos problemas requieren la transformación de sistemas tecnológicos enteros, más allá de impulsar ideas de innovación orientadas al sector privado como solución a los problemas sociales.

De esta manera, surge un nuevo concepto, el de innovación social (Van der Have y L. Rubalcaba, 2016). Así, la innovación social se refiere a la innovaciónen las relaciones, prácticas y estructuras sociales (como los modelos comerciales, las prácticas de producción, la financiación y la prestación de servicios públicos) principalmente encaminadas a satisfacer las necesidades sociales y mejorar el bienestar humano ${ }^{10}$.

La mayoría de las innovaciones sociales se inician desde la base, a través de actividades empresariales llevadas a cabo por organizaciones y agentes de la sociedad civil y el tercer sector (como cooperativas, asociaciones y fundaciones). Por ejemplo, los modelos de producción de comercio justo a escala mundial asocian los movimientos sociales, los productores, las empresas convencionales y sus correspondientes normas de maneras novedosas. Las empresas e iniciativas sociales, como, por ejemplo, el uso de iniciativas de comercio justo para vender productos derivados de la producción de conocimientos ancestrales y demás cuestiones, son ejemplos indicativos de esto.

De esta manera, la innovación orientada a estos objetivos sociales, puede tener un apoyo en los incentivos que brinda la protección de la propiedad intelectual, por cuanto, esta permite captar el valor de estos desarrollos e incluso fijar prioridades de protección a aquellas tecnologías que favorezcan el medioambiente, la transparencia en el reparto de 
recursos y demás cuestiones atinentes a brindar una adecuada protección a aquellos que desarrollan y permiten producir mejoras tecnológicas desde colectivos de individuos.

\section{Conclusiones}

Los derechos de propiedad intelectual son sin duda relevantes para el desarrollo, como lo demuestra la bioprospección. El bioprospector espera recuperar la inversión realizada, y para ello los beneficios económicos que supone la propiedad intelectual no sólo son un incentivo, sino una condición sine qua non. La participación en los beneficios generados de las ventas del producto, al depender éstos de una efectiva protección de derechos, constituye por ello no un obstáculo sino un incentivo para que los Estados establezcan y mantengan un régimen de protección a la propiedad intelectual, tanto en su propia jurisdicción como en la esfera internacional.

La cuestión a resolver no es por tanto si es necesario o no un sistema de protección de la propiedad intelectual, sino qué clase de sistema conviene más y cuáles son los límites que se deben fijar, particularmente en lo que respecta a la definición de lo que entra en el estado de la técnica, y por tanto, el sentido que se le debe dar al requisito de novedad en la invención/variedad vegetal. Una de las cuestiones técnicas que sin duda conviene analizar es la viabilidad de exigir la divulgación de la fuente de la que provienen los recursos genéticos (Estado de origen) y los conocimientos tradicionales (comunidad involucrada) utilizados. Para ello, los acuerdos de prospección pueden funcionar como prueba de un acceso legítimo (bajo consentimiento fundamentado previo y en condiciones mutuamente acordadas) a los recursos genéticos y conocimientos asociados en las solicitudes de patentes y de derechos de obtentor.

Sin embargo, otras soluciones creativas son posibles; para garantir no sólo los derechos de las comunidades originarias y sus expresiones culturales de forma de vida, sino también, comprender que la organización y la protección de esto puede proveer una herramienta efectiva de desarrollo sustentable y económico a los países; por medio de la cooperación, solidaridad y desarrollo de estrategias que integren el respeto cultural por los conocimientos culturales y su aprovechamiento económico.

\section{Notas}

1. A tales fines para contrastar visiones ver: NOZICK, Robert, Anarchy, State and Utopia, Basick Books, inc. Harper Torchbooks, New York, 1974 [trad. cast. Rolando Tamayo, Anarquía, Estado y Utopía, FCE, Mexico, 1988], p. 235-238.

2. A tales fines ver: ASENSIO, Pedro A. De Miguel, "Derechos fundamentales y observancia de los derechos de autor en la Unión Europea”, en la obra: El derecho internacional en el mundo multipolar del siglo XXI,Iprolex, Madrid, 2013, p. 713-738

3. Ver para más detalle: Necesidades y expectativas en materia de propiedad intelectual de los titulares de conocimientos tradicionales - Informe de la OMPI relativo a las mi- 
siones exploratorias sobre propiedad intelectual y conocimientos tradicionales, OMPI, 2001, 25.

4. La representación en la vida del pueblo refiere a que los vínculos con el territorio y con los recursos biofísicos por parte de las comunidades que conservan, en lo esencial, su cosmovisión e integridad cultural, con lo cual promueven relaciones de respeto hacia la naturaleza y de solidaridad hacia los demás miembros de la comunidad originaria y pueblo indígena. Así los conocimientos tradicionales forman parte de idiosincrasia de sus titulares y traducen parte de su concepción de vida.

5. Entre otros: Convenio sobre la Diversidad Biológica, adoptado en Río de Janeiro el 5 de junio de 1992; puede consultarse en http://www.biodiv.org, Tratado Internacional sobre Recursos Genéticos para la Alimentación y la Agricultura, aprobado en Roma el 3 de noviembre del 2001 por la Resolución 3/2001 de la Conferencia de la FAO; puede obtenerse en http://www.fao.org, Protocolo de Cartagena sobre Seguridad de la Biotecnología del Convenio sobre la Diversidad Biológica, adoptado en Montreal el 29 de enero de 2000; disponible en http://www.biodiv.org y Acuerdo sobre los Aspectos de los Derechos de Propiedad Intelectual relacionados con el Comercio (ADPIC), del 15 de abril de 1994; reproducido en Los resultados de la Ronda Uruguay de negociaciones comerciales multilaterales. Los textos jurídicos, Ginebra, Secretaría del GATT, 1994, pp. 381-419; así como en http://www.wto.org.

6. Ver: Declaración de las Naciones Unidas sobre los derechos de los pueblos indígenas, artículo31, inc. 1, la cual fue adoptada en Nueva York el 13 de septiembre de 2007 durante la sesión 61 de la Asamblea General de las Naciones Unidas.

7. Ver los estudios de: J. Terrence MCCABE, Turkana Pastoralism: A case against the Tragedy of the Commons, Human Ecology, 18 (1), 1994, 81 a 103 y MCCAY AND ACHESON, Human Ecology of the Commons, University of Arizona, Tucson, 1994. Ambos citados por: Gabriel R. Nemogá SOTO, Régimen de propiedad sobre recursos genéticos y conocimiento tradicional, Editorial Universidad Nacional de Colombia, Bogotá, 2004, 5

8. Dutfield, G., Intellectual Property Rights, Trade and Biodiversity, London, IUCN, Earthscan Publications Ltd., 2000, p. 45, quien también identifica otras causas de la pérdida de biodiversidad más directas, como los cambios demográficos, las disparidades en la distribución y control de la tierra, las presiones de las industrias agroquímica y de semillas, las políticas de apoyo a la agricultura extensiva y la falta de respeto a los conocimientos tradicionales.

9. Cfr. Cripps, Y., "Aspects of Intellectual Property in Biotechnology: Some European Legal Perspectives” en Guruswamy, L. D. y McNeely, J.A. (eds.), op. cit., p. 328, para quien: "Although intellectual property systems have implications for biodiversity, the granting or witholding of patents will not constitute an adequate response to the social, moral and environmental dimensions of the task of preserving the world's natural resources".

10. Ver informe: Nuevos criterios de innovación para apoyar la aplicación de los Objetivos de Desarrollo Sostenible, Comisión de Ciencia y Tecnología para el Desarrollo, 2017, UN. 


\title{
Referencias Bibliográficas
}

\section{Libros y artículos de revistas jurídicas}

Buitrago, H. (2017). La propiedad intelectual y el uso sostenible de los recursos naturales y biodiversidad. Colombia. Revista Colombiana de Biotecnología. Vol. XIX No. 2.

Cripps, Y. (2000). “Aspects of Intellectual Property in Biotechnology: Some European Legal Perspectives” en Guruswamy, L.D. y McNeely, J.A. (eds.)

Dutfield, G. (2000). Intellectual Property Rights, Trade and Biodiversity, London, IUCN, Earthscan Publications Ltd.

Downes, y G. Wiser. (2002). Intellectual Property Rights and National Access and BenefitSharing Measures, en Laird, S. (ed.), Biodiversity and Traditional Knowledge. Equitable Partnerships in Practice, London, Earthscan/People and Plants Conservations Series Hardin, Garrett. (1968). The Tragedy of the Commons, Science 162, New York.

Heller, Michael A. y Rebeca S. Eisenberg. (1998). Can patents deter innovation? The Anticommons in Biomedical Research. En: Science. Volumen 80. Número 5364

Mazzucato. (2013). The Entrepreneurial State: Debunking Public vs. Private Sector Myths Anthem Press, Londres.

Nakayima, Douglas. (1998). Conceptualizing nature: the cultural nature of resources management, UNESCO

Ruiz, Manuel. (1999). Protecting indigenous peoples knowledge: a legislative perspective from Perú. Sociedad Peruana de Derecho Ambiental.

Sandel, Michael J. (1982). Liberalism and the Limits of Justice, Cambridge University Press.

Soto, Hernando de, en su libro, El Misterio del Capital, ¿Por qué el capitalismo triunfa en occidente y fracasa en el resto del mundo?, en: http://www.elcato.org/special/friedman/ desoto/cap1_misteriodelcapital.html (Acceso el 02/2/2019)

Van Der Have y L. Rubalcaba, 2016, Social innovation research: An emerging area of innovation studies? Research Policy 45(9):1923 a 1935.

Zamudio, Teodora. (2013). Protección de los derechos inherentes a los proveedores de los insumos genéticos y culturales asociados de las invenciones patentables. Armonización de los derechos de propiedad intelectual y los derechos emergentes del Convenio de Diversidad Biológica. Buenos Aires, Argentina.

\begin{abstract}
The concept of intellectual property is analyzed and harmonized with the concept of sustainability. A concrete example of possible regulation is traditional knowledge. It is demonstrated that it is possible to harmonize the economic use of intellectual property with an innovative impact on social development and the cultural environment.
\end{abstract}

Keywords: intellectual property - development - traditional knowledge.

Resumo: O conceito de propriedade intelectual é analisado e harmonizado com o conceito de sustentabilidade. Em particular, é mostrado um exemplo concreto de possí- 
vel regulamentação: o conhecimento tradicional e, com ele, é demonstrado que é possível harmonizar o uso econômico da propriedade intelectual com um impacto inovador no desenvolvimento social e no ambiente cultural.

Palavras chave: propriedade intelectual - desenvolvimento - conhecimento tradicional.

[Las traducciones de los abstracts fueron supervisadas por el autor de cada artículo] 